\title{
Procedimentos invasivos em usuários em internação domiciliar
}

\author{
Invasive procedures on patients under \\ home-care
}

\section{Thaís Marques Moura' ${ }^{10}$ Gilmara Ribeiro Santos Rodrigues ${ }^{2}(\mathbb{0}$ Victor Fernando Alves $\mathrm{Neves}^{3}$ () Gleide Regina de Sousa Almeida Oliveira ${ }^{4}$ ()}

\footnotetext{
${ }^{1}$ Autora para correspondência. Escola Bahiana de Medicina e Saúde Pública (Salvador). Bahia, Brasil. thaismoura16.1@bahiana.edu.br ${ }^{2-3}$ Escola Bahiana de Medicina e Saúde Pública (Salvador). Bahia, Brasil. gilmararodrigues@bahiana.edu.br, victor_nevesba@hotmail.com ${ }^{4}$ SOS VIDA (Salvador). Bahia, Brasil. gleideenf@gmail.com
}

RESUMO | OBJETIVO: Descrever os procedimentos invasivos predominantes nos usuários em internação domiciliar. MÉTODO: Estudo quantitativo descritivo retrospectivo a partir de um banco de dados de pacientes que estiveram internados em uma unidade privada de assistência domiciliar na cidade de Salvador, BA, Brasil. A amostra foi constituída de 110 prontuários. Para a coleta de dados foi utilizado um formulário com as seguintes variáveis: dados sociodemográficos, suporte terapêutico; suporte ventilatório e terapia nutricional. Os dados foram analisados em frequências absolutas e relativas. RESULTADOS: Observou-se a ocorrência de 152 procedimentos invasivos nos 110 pacientes. Dentre os suportes terapêuticos, predominou o cateter central de inserção periférica $(92,0 \%)$, seguido do cateter venoso central $(33,0 \%)$. Em relação ao suporte ventilatório, traqueostomia foi a mais frequente $(25,0 \%)$, e quanto à terapia nutricional prevaleceu a gastrostomia $(46,1 \%)$. Dentre os dispositivos de eliminação vesical, a sonda vesical de demora apresentou percentual de 10,5\%. CONCLUSÃO: os procedimentos invasivos mais recorrentes nos pacientes na atenção domiciliar foram a PICC (Cateter Central de Inserção Periférica), a traqueostomia e a gastrostomia. Delimitar o perfil de procedimentos realizados na atenção domiciliar possibilita a construção de protocolos relacionados a estes procedimentos, criação de estratégias de cuidados no domicílio e de prevenção de eventos adversos e orientação dos pacientes e familiares/cuidadores.

DESCRITORES: Assistência domiciliar. Serviços de assistência domiciliar. Desospitalização.
ABSTRACT | OBJECTIVE: To describe the predominant invasive procedures on patients under home-care. METHOD: A retrospective quantitative and descriptive study from databanks of patients cared for through a private home-care unit in the city of Salvador, BA, Brazil. The sample comprised 110 medical records. For data collection a form was used with the following variables: sociodemographic data, therapeutic support, ventilatory support and nutritional therapy. The data was analyzed in absolute and relative frequencies. RESULTS: The occurrence of 152 invasive procedures was observed on the 110 patients. Within the therapeutic supports, predominance was for peripherally inserted central catheter (PICC) (92.0\%), followed by central venous catheters (33.0\%). In relation to ventilatory support, tracheostomy was the most frequent $(25.0 \%)$, and with reference to nutritional therapy, prevalence was for gastrostomy (46.1\%). Within the devises for bladder elimination, the indwelling urinary catheter presented a percentage of $10.5 \%$. CONCLUSION: The most recurrent invasive procedures in patients under home care were the PICC (peripherally inserted central catheters, tracheostomy and gastrostomy. Determining the profile of procedures performed in home-care permits the construction of protocols related to such procedures, creation of homecare health strategies and the prevention of adverse events and guidance for patients and family members/caregivers.

DESCRIPTORS: Home-care. Home-care services. Dehospitalization. 


\section{Introdução}

No modelo hegemônico hospitalocêntrico, os cuidados realizados pela equipe multiprofissional vão desde atividades de vida diária (AVD's) até procedimentos invasivos, que estão diretamente associados às Infecções Relacionadas à Assistência à Saúde (IRAS's). A constante exposição a procedimentos invasivos resulta em malefícios para os usuários, como a internação prolongada, elevado risco de mortalidade e desenvolvimento de infecções multirresistentes, que ocasionam período de altos custos hospitalares e a dispersão de microrganismos multirresistentes' .

A atenção domiciliar (AD) surge, nesse contexto, como uma possibilidade para minimizar esses agravos. O Ministério da Saúde define $A D$, como sendo os cuidados prestados em domicílio visando à prevenção e tratamento da doença, assim como a reabilitação, paliação e promoção da saúde, garantindo a continuidade de cuidados. A AD é uma modalidade de atenção à saúde integrada as Redes de Atenção à Saúde (RAS) ${ }^{2-3}$.

Os Programas de Atenção Domiciliar (PADs) estão vinculados às Unidades de Pronto Atendimento (UPAs), previstos como componentes da Rede de Atenção às Urgências e são organizados para atender a pacientes em agudização de algum quadro clínico cuja internação hospitalar seja indicada, mas passível de ser evitada por meio de $\mathrm{AD}^{3}$.

A assistência domiciliar desponta como uma categoria que possibilita um novo modelo de Atenção à Saúde no Brasil, contribuindo para a redução de custos hospitalares e principalmente para a perspectiva da desospitalização, favorecendo uma assistência segura e humanizada do profissional de Enfermagem. No Brasil, o público assistido pela AD é predominantemente composto por pessoas idosas, em cuidado paliativos, com patologias pulmonares crônicas, dependentes de oxigenioterapia e crianças prematuras ${ }^{4}$.
O modelo de assistência domiciliar é percebido como um esforço de mudança na organização dos serviços de saúde, que busca superar o modelo assistencial centrado em cuidados hospitalares, embora os mesmos sejam imprescindíveis em situações específicas ${ }^{5}$. Nesse modelo, destaca-se a internação domiciliar, definida como uma infraestrutura minimizada do hospital ao domicílio, sendo uma estratégia de desospitalização. Retrata uma alternativa segura e eficaz para a atenção a pacientes específicos, clinicamente estáveis, mas que requerem cuidados singulares e diários do profissional de saúde ${ }^{6}$.

Nessa modalidade de assistência muitas vezes se faz necessária à realização de procedimentos invasivos. Muitas ações realizadas em organizações hospitalares, como utilização de dispositivos de acesso venoso, sonda de eliminação vesical, terapia de suporte ventilatório e nutrição enteral podem ser realizadas no domicílio, desde que, avaliados e controlados os riscos para o paciente, família e o profissional de saúde que a realiza.

Foi realizada busca nas bases de dados BVS, Portal CAPES, LILACS e PUBMED, combinando o descritor "procedimentos invasivos" com "assistência domiciliar", "serviços de assistência domiciliar", "desospitalização" e "home care", que constatou que não há pesquisas que retratam particularmente esta temática.

Dessa forma, é relevante o propósito de investigar os procedimentos invasivos predominantes em usuários em internação domiciliar cuja finalidade está relacionada a obtenção de informações que subsidiem estratégias da melhor qualidade da prestação dos cuidados de enfermagem no domicílio, favorecendo assim, a atuação de forma segura e humanizada do profissional de Enfermagem. Para tanto, delimitamos como objetivo descrever os procedimentos invasivos predominantes em usuários em internação domiciliar. 


\section{Método}

Estudo retrospectivo quantitativo descritivo com utilização de dados secundários, a partir do banco de dados da pesquisa maior intitulada "Perfil sociodemográfico e clínico de usuários assistidos na atenção domiciliar", sob número do CAAE 99850418.6.0000.5544. A coleta de dados foi realizada em outubro e novembro de 2019.

No banco de dados constavam dados sociodemográficos e clínicos de pacientes que estiveram internados em uma unidade privada de assistência domiciliar na cidade de Salvador, BA, Brasil. Este serviço foi escoIhido por ser o pioneiro no estado da Bahia a prestar cuidados domiciliares a pacientes crônicos e por admitir pacientes com doenças crônicas, com quadro clínico estável e em condições de prosseguir com o tratamento em domicílio.

O banco de dados era constituído com informações de 226 prontuários de pacientes com história de internação domiciliar. A amostra foi composta por 110 prontuários de pacientes que atendiam aos seguintes critérios de inclusão: adultos e idosos, ambos os sexos, que passaram por internamento hospitalar em uma unidade de terapia intensiva e foram admitidos na AD entre janeiro de 2017 a julho de 2018. Foram excluídos os prontuários de pacientes que ainda se encontrem em internação domiciliar, os que não tiverem todas as informações pertinentes e o histórico admissional de enfermagem e médico preenchidos.

A delimitação temporal não consistiu um viés para a desatualização dos dados encontrados porque os procedimentos invasivos predominantes em usuários em internação domiciliar não apresentam mudanças constantes.
Foi utilizado um formulário para coleta de dados que continha as seguintes variáveis: dados sociodemográficos (sexo, idade, espiritualidade/religião, ocupação e escolaridade), suporte terapêutico (acesso venoso central; o acesso venoso periférico; a punção venosa intermitente; a hipodermóclise), suporte ventilatório (traqueostomia, ventilação mecânica e aspiração de vias aéreas), terapia nutricional (gastrostomia, sonda nasoenteral, jejunostomia, ileostomia e nutrição parenteral total) e de eliminação vesical (sondagem uretral intermitente e a vesical de demora). Os dados depois foram tabulados no programa Excel ${ }^{\circledR}$ e após analisados foram apresentados em frequências $a b$ solutas e relativas.

A pesquisa obedeceu aos princípios da Resolução $\mathrm{N}^{\circ} 466 / 12$ que se refere aos aspectos sobre pesquisa envolvendo seres humanos abordando os quatro referenciais básicos da bioética: autonomia, não maleficência, beneficência e justiça, preservando em caráter confidencial todas as informações coletadas e analisadas no estudo. Sendo assim, a pesquisa foi aprovada pelo Comitê de Ética da Escola Bahiana de Medicina e Saúde Pública, Salvador, BA, sob o número de parecer CAAE 99850418.6.0000.5544. Não se fez necessária à assinatura do TCLE por se tratar de uma pesquisa em dados secundários e por ser impossível o acesso aos pacientes, por terem tido alta do tratamento domiciliar desde a realização da pesquisa maior.

\section{Resultados}

Quanto às características sociodemográficas, observou-se que $54,5 \%$ era do sexo masculino, $(74,6 \%)$ católicos, (72,8\%), com mais de 60 anos de idade, (61\%) aposentados e (45,5\%), com o ensino médio completo (Tabela 1). 
Tabela 1. Características sociodemográficas de pacientes da atenção domiciliar, Salvador, BA, Brasil, 2017-2018. (N=110)

\begin{tabular}{|c|c|c|}
\hline Variáveis & $\mathbf{N}$ & $\%$ \\
\hline \multicolumn{3}{|l|}{ Sexo } \\
\hline Feminino & 50 & 45,5 \\
\hline Masculino & 60 & 54,5 \\
\hline \multicolumn{3}{|l|}{ Faixa etária } \\
\hline 25-30 anos & 1 & 0,9 \\
\hline $31-40$ anos & 12 & 10,9 \\
\hline 41-50 anos & 4 & 3,6 \\
\hline $51-60$ anos & 13 & 11,8 \\
\hline$>60$ anos & 80 & 72,8 \\
\hline \multicolumn{3}{|l|}{ Profissão } \\
\hline Aposentado & 67 & 61,0 \\
\hline Autônomo & 15 & 13,6 \\
\hline Do lar & 16 & 14.5 \\
\hline Outros & 12 & 10.9 \\
\hline \multicolumn{3}{|l|}{ Escolaridade } \\
\hline Ensino fundamental incompleto & 24 & 22,0 \\
\hline Ensino fundamental completo & 10 & 9,0 \\
\hline Ensino médio incompleto & 3 & 2,7 \\
\hline Ensino médio completo & 50 & 45,5 \\
\hline Ensino superior incompleto & 3 & 2,7 \\
\hline Ensino superior completo & 20 & 18,1 \\
\hline \multicolumn{3}{|l|}{ Espiritualidade/religião } \\
\hline Católico & 82 & 74,6 \\
\hline Evangélico & 16 & 14,5 \\
\hline Espírita & 7 & 6,4 \\
\hline Ateu & 5 & 4,5 \\
\hline
\end{tabular}

Fonte: Os autores (2020).

Foram considerados como procedimentos invasivos, os suportes terapêuticos, ventilatórios, tipo de terapia nutricional e de eliminação vesical (tabela 2). Vale ressaltar que os somatórios dos percentuais podem não corresponder à $100 \%$ devido alguns pacientes fazer uso de mais de um dispositivo invasivo, sendo realizados um total de 152 procedimentos invasivos.

A Tabela 2 reúne a descrição dos procedimentos invasivos utilizados por pacientes em internação domiciliar. Ao considerar os suportes terapêuticos, observou-se que os dispositivos e procedimentos mais utilizados estavam relacionados à terapêutica medicamentosa venosa via Cateter Central de Inserção Periférica (PICC) (92,0\%), seguido pelo cateter venoso central (33\%). Dentre os pacientes que usavam algum tipo de dispositivo para eliminação vesical, 10,5\% usavam sonda vesical de demora e 4,0\% realizavam a sondagem vesical de alívio.

Em relação ao suporte ventilatório, predominou o uso da traquestomia $(25,0 \%)$, não sendo encontrado paciente em uso de entubação. Quando avaliado a utilização de terapia nutricional, verificou-se que $46,1 \%$ dos pacientes estavam sendo alimentados pela gastrostomia e 11,84\% via sonda nasoenteral (Tabela 2). 
Tabela 2. Distribuição dos procedimentos invasivos utilizados por pacientes em internação domiciliar, Salvador, BA, Brasil, 2019

\begin{tabular}{lcc}
\hline Descrição & $\mathbf{n}$ & $\%$ \\
\hline Suporte terapêutico & & 92,0 \\
PICC * & 46 & 8,0 \\
Port a cath & 4 & 33,0 \\
Acesso Venoso Central (CVC) & 50 & 17,8 \\
Acesso Venoso Periférico (AVP) & 27 & 3,3 \\
Hipodermóclise & 5 & 10,5 \\
& & 4,0 \\
Eliminação Vesical & 16 & 25,0 \\
Sonda Vesical de Demora (SVD) & 6 & 6,6 \\
Sonda Vesical de Alívio (SVA) & & 12,5 \\
Suporte ventilatório & 38 & \\
Traqueostomia & 10 & 11,8 \\
Ventilação Mecânica (VM) & 19 & 1,3 \\
Aspiração de Vias Aéreas (AVA) & & 1,3 \\
Terapia nutricional & 70 & 1,3 \\
$\quad$ Gastrostomia & 18 & \\
Sonda Nasoenteral (SNE) & 2 & 2 \\
Jejunostomia & 2 & \\
Ileostomia & & \\
Nutrição Parenteral Total (NPT) & & \\
\hline *PICC - Cateter Central de Inserção Periférica &
\end{tabular}

*PICC - Cateter Central de Inserção Periférica

Fonte: Os autores (2020).

A tabela 3 apresenta a quantidade de dispositivos utilizados por um mesmo paciente, onde se observa que a maioria $(55,9 \%)$ usava apenas um dispositivo.

Tabela 3. Quantidade de dispositivos invasivos utilizados por pacientes na internação domiciliar, Salvador, BA, Brasil, 2019

\begin{tabular}{ccc}
\hline Quantidade de dispositivos & $\mathbf{N}$ & \% \\
\hline 1 & 85 & 55,9 \\
2 & 35 & 23,0 \\
3 & 19 & 12,5 \\
4 & 8 & 5,3 \\
5 & 4 & 2,6 \\
\hline
\end{tabular}

Fonte: Os autores (2020). 


\section{Discussão}

Os pacientes alvos da análise deste estudo são caracterizados predominantemente pela faixa etária acima de 60 anos. Este achado é importante a partir do conhecimento que as pessoas idosas, quando comparado com o jovem, são mais susceptíveis aos efeitos adversos dos procedimentos invasivos, especialmente à infecção, em razão de alterações fisiológicas do envelhecimento, declínio da resposta imunológica e a presença de doenças concomitantes ${ }^{7}$.

Como no contexto nacional e internacional há escassez de estudos sobre procedimentos invasivos utilizados por usuários em internação domiciliar, não foi possível comparar esses resultados com várias outras investigações.

Dentre os achados desta pesquisa, o PICC foi o dispositivo de suporte terapêutico mais utilizado, o que se justifica pelas vantagens da implantação: como o baixo risco de infecção; ser um acesso venoso eficaz; poder ser realizado à beira leito; ter longo tempo de permanência ( 2 anos e 6 meses) e ter maior segurança na administração medicamentosa8. O PICC tem indicação segura para os pacientes em cuidados paliativos visto que há a vulnerabilidade e sofrimentos físico e psicológico frente ao processo do adoecimento, que contribuem significativamente para a qualidade de vida. As veias mais indicadas são a basílica e a cefálica porque possuem o trajeto menor até a veia cava superior $^{9-11}$.

Enquanto que o cateter venoso central (CVC) no domicílio não é tão vantajoso, por não permitir uso intermitente e possuir curta duração ${ }^{11}$. Outra desvantagem refere-se ao uso domiciliar impróprio tanto pelo fato de deslocamento do dispositivo e maior risco de infecção ${ }^{12}$. Embora o CVC guiado por ultrassom seja uma perspectiva, ainda não é adotado absolutamente ${ }^{13}$.

Em um estudo realizado na Índia, a conexão entre inúmeros fatores quanto às adversidades envolvendo a inserção do CVC; $15 \%$ dos pacientes apresentaram complicações associadas ao CVC, dentre essas complicações, $19 \%$ são mecânicas, $26 \%$ infecciosas e $26 \%$ tromboembolismo ${ }^{14}$. Segundo estudos nos EUA, afirmam que as complicações relacionadas à punção arterial acidental também são muito frequentes, 15\% das tentativas acarretam problemas ${ }^{15}$.
A despeito do Port a Cath ser um dispositivo amplamente utilizado na oncologia, o perfil de muitos pacientes que recebem cuidados em domicílio, correspondeu apenas $8,0 \%$ da via utilizada para terapia medicamentosa venosa. É um dos modelos de cateter de longa permanência, totalmente implantável e que possui vantagens como maior durabilidade, menor risco de infecção e nenhuma exteriorização do seguimento do conjunto ${ }^{11,16}$.

O acesso venoso periférico representou $17,8 \%$ dos tipos de dispositivos usados por pacientes internados em domicílio. É evidenciado que o acesso periférico é um procedimento de baixo risco, custo reduzido e curta durabilidade, por mais que seja utilizado em pacientes internados. É necessária a troca contínua para a prevenção da infecção de corrente sanguínea e de extravasamento de medicações, além de requerer punções frequentes. Seu uso para os pacientes que fazem tratamento quimioterápico não é indicado, porque as soluções vesicantes podem provocar complicações tecidual ${ }^{11}$.

A Hipodermóclise também denominada de terapia subcutânea, com 3,3\%, é outra alternativa para pacientes em cuidados paliativos no ambiente domiciliar. De acordo com o Instituto Nacional de CâncerINCA, trata-se da infusão de fluidos isotônicos e/ ou medicações pela via subcutânea. A vantagem em se utilizar esse procedimento invasivo está relacionada à disponibilidade sérica da medicação quanto à sua liberação prolongada, facilitando por mais tempo o alívio dos sintomas e a diminuição das complicações vasculares e sistêmicas ${ }^{17}$.

A Hipodermóclise é a segunda via de escolha, quando a via oral e endovenosa é inviável, possibilitando controle, conforto dos sintomas e qualidade de vida para esses pacientes. Apesar disso, ainda é pouco utilizada, mesmo sendo uma técnica segura, com complicações irrelevantes, de fácil acesso, aplicação e manuseio, além de utilizar um dispositivo de baixo custo e causar menos dor, em comparação ao acesso venoso periférico. Em relação às desvantagens, não permite a administração de grandes quantidades de volumes, tem limitação em relação ao uso de alguns medicamentos e de eletrólitos ${ }^{17}$. 
Durante a assistência domiciliar o acesso deverá ser reavaliado em dias alternados, podendo permanecer de 5 a 7 dias. Na infusão contínua o volume diário total não deve ultrapassar $3000 \mathrm{ml}$. O débito da perfusão pode variar entre 1 a $8 \mathrm{ml} / \mathrm{min}$ dependendo do volume e da solução infundida ${ }^{18}$.

Dentre os procedimentos invasivos utilizados no cuidado prestado em domicílio, estão os dispositivos de eliminação vesical - sonda vesical de demora e cateter vesical de alívio, procedimentos invasivos realizados extensivamente pela enfermeira na atenção domiciliar. A técnica asséptica deve ser seguida rigorosamente para garantir a segurança e prevenir as adversidades, dentre elas, a infecção do trato urinário (ITU). Além disso, constata-se a importância da tomada de decisão da enfermeira, ao utilizar esses dispositivos invasivos, sendo uma avaliação imprescindível e fundamentada de acordo com a condição clínica do paciente, para a precaução de futuras complicações ${ }^{19-20}$.

Quanto à traqueostomia ter sido a terapêutica de suporte ventilatório mais utilizada, o percentual encontrado nesta pesquisa foi semelhante ao encontrado por estudo realizado com paciente em internação domiciliar no Rio Grande do Sul $(24,6 \%)^{21}$ e menor do que o encontrado no estudo realizado igualmente com banco de dados de 859 prontuários de instituições que prestam Serviço de Assistência Domiciliar do município de Maceió $(9,0 \%)^{22}$.

A traqueostomia possui vantagens como maior conforto e menor vulnerabilidade a infecções, além de ser uma opção viável para facilitar a extubação e reduzir custos hospitalares por permitir a alta hospitalar para o domicílio. De acordo com a literatura a traqueostomia é destinada para pacientes com afecções graves do sistema respiratório e diagnosticados com disfunção pulmonar e da musculatura ventilatória, sendo uma interface para a ventilação ser mais efetiva, possibilitando a transferência com o suporte ventilatório domiciliar ${ }^{23}$.

É constatado ainda que a ventilação mecânica já é considerada uma prática corriqueira na assistência à saúde, pois houve aumento do número e da complexidade dos quadros clínicos dos pacientes, portanto é necessária uma avaliação criteriosa quanto ao uso desse dispositivo invasivo. Sendo importante ressaltar a necessidade da manutenção das vias aéreas permeáveis e da aspiração, principalmente quando os pacientes estão acamados e em uso de suporte ventilatório ${ }^{23}$.

A terapia nutricional domiciliar refere-se à assistência nutricional e clínica ao paciente em domicílio com o objetivo de recuperar ou manter o nível máximo de saúde, funcionalidade e comodidade do paciente ${ }^{24}$. Chama a atenção a frequência de pacientes fazer uso de terapia nutricional por gastrostomia. Este achado pode ser devido a um longo tempo de internamento em domicílio com diversas tentativas de manutenção de sonda enteral sem êxito ou devido o perfil dos pacientes que geralmente são idosos que requerem o máximo de absorção dos nutrientes. No estudo de Carnaúba e colaboradores ${ }^{22}, 15,6 \%$ dos pacientes se alimentam por via gastrostomia, 15,3\% por sonda naso-enteral (SNE) ou naso-gástrica (SNG).

No domicílio, a enfermeira tem que orientar os pacientes e família quanto à necessidade e como manter a sonda pérvia, detectar problemas de obstrução, vazamentos, exteriorização, higienização e o preparo e administração da dieta.

No ambiente domiciliar, é essencial a escolha do dispositivo ideal e adequado a cada paciente, visando prevenir e diminuir o risco de complicações. É preciso também avaliar o preparo e as condições da família para cuidar do paciente em domicílio e a necessidade ou não de um profissional de saúde no domicílio. Além disso, nos casos possíveis, estimular a autonomia do próprio paciente, orientando-o e treinando-o em relação aos procedimentos e dispositivos.

Normalmente, um mesmo paciente faz uso de mais de um dispositivo simultâneo, o que ficou evidenciado em quase metade dos pacientes desta pesquisa, diferente do contexto hospitalar no qual maioria dos pacientes internados, usarem apenas um dispositivo invasivo (55,9\%). Constatou-se em outro estudo com 54 pacientes no regime de internação domiciliar, que $31,5 \%$ realizaram traqueostomia e gastrostomia concomitantemente e $3,7 \%$ gastrostomia e acesso venoso periférico simultaneamente ${ }^{25}$.

O estudo apresenta como limitação ter sido realizada em apenas um serviço de atenção domiciliar, impedindo que os resultados possam ser generalizáveis para todos e quaisquer pacientes em internação domiciliar. Todavia, os mesmos se tornam uteis para pacientes internados em unidades de saúde com o mesmo perfil do lócus de pesquisa. 


\section{Conclusão}

Este estudo alcançou o objetivo de descrever os procedimentos invasivos mais recorrentes nos pacientes na atenção domiciliar, que foram a PICC, a traqueostomia e a gastrostomia. Delimitar o perfil de procedimentos realizados na atenção domiciliar, possibilita a construção de protocolos relacionados a estes procedimentos e a criação de estratégias de cuidado no domicilio, visando a prevenção de eventos adversos e a orientação dos pacientes e familiares/cuidadores.

Destaca-se a necessidade de novas pesquisas sobre o objeto de estudo no contexto domiciliar com diferentes metodologias, principalmente pesquisa de campo, visto que esse tipo de serviços e usuários estão em expansão.

\section{Contribuições dos autores}

Moura TM participou da concepção, delineamento, análise dos dados da pesquisa, interpretação dos resultados e redação do artigo científico. Rodrigues GRS participou da concepção, delineamento, busca e análise dos dados da pesquisa, interpretação dos resultados, redação do artigo científico. Neves VFA e Oliveira GRSA participaram da coleta de dados da pesquisa e aprovaram a versão final do manuscrito.

\section{Conflitos de interesses}

Nenhum conflito financeiro, legal ou político envolvendo terceiros (governo, empresas e fundações privadas, etc.) foi declarado para nenhum aspecto do trabalho submetido (incluindo, mas não se limitando a subvenções e financiamentos, participação em conselho consultivo, desenho de estudo, preparação de manuscrito, análise estatística, etc.).

\section{Referências}

1. Carvalho DP, Toso BRGO, Viera CS, Garanhani ML, Rodrigues RM, Ribeiro LFC. Ser cuidador e as implicações do cuidado na atenção domiciliar. Texto contexto - enferm. 2015;24(2):450-8. doi: 10.1590/0104-07072015000782014

2. Ministério da Saúde (Brasil). Portaria $n^{\circ} 825$, de 25 de abril de 2016. Redefine a Atenção Domiciliar no âmbito do Sistema Único de Saúde (SUS) e atualiza as equipes habilitadas. Diário Oficial da União [da] República Federativa do Brasil. 2016 abr 26;153(78 Seção 1):33-8. Disponível em: http://www.in.gov.br/materia/-/ asset_publisher/Kujrw0TZC2Mb/content/id/22685962/do1-201604-26-portaria-n-825-de-25-de-abril-de-2016-22685827
3. Ministério da Saúde. Secretaria de Atenção à Saúde. Departamento de Atenção Básica. Caderno de atenção domiciliar / Ministério da Saúde, Secretaria de Atenção à Saúde, Departamento de Atenção Básica. [Internet]. 2012. [acesso em 18 nov. 2018]. Disponível em: http://189.28.128.100/dab/docs/ publicacoes/geral/cad_vol1.pdf

4. Nery BLS, Favilla FAT, Albuquerque APA, Salomon ALR. Characteristics of home care services. Journal of Nursing UFPE on line. 2018;12(5):1422-9. doi: 10.5205/1981-8963-v12i5a23060 4p1422-1429-2018

5. Savassi LCM. Os atuais desafios da Atenção Domiciliar na Atenção Primária à Saúde: uma análise na perspectiva do Sistema Único de Saúde. Rev Bras Med Fam Comunidade. 2016;11(38):112. doi: $10.5712 / \mathrm{rbmfc} 11(38) 1259$

6. Schwonke CRGB, Silva SJR, Casalinho ALD'A, Santos CM, Vieira FP. Internação domiciliar: reflexões sobre a participação do cuidador/família/enfermeiro no cuidado. Ensaios e Ciência: Ciências Biológicas, Agrárias e da Saúde. 2008;12(1):77-90.

7. Toffoletto MC, Barbosa RL, Andolhe R, Oliveira EM, Janzantte DA, Padilha KG. Fatores relacionados à ocorrência de eventos adversos em pacientes idosos críticos. Rev Bras Enferm. 2016;69(6):1039-45. doi: 10.1590/0034-7167-2016-0199

8. Reis NSP, Santos MFG, Leite DC, Gomes HF, Peres EM, Perez Junior EF. Implantação de cateter central de inserção periférica por enfermeiros em adolescentes. Cogitare Enfermagem. 2019;24(esp.):1-11. doi: 10.5380/ce.v24i0.55639

9. Alcântara DC, Peregrino AAF, Jesus CS, Siqueira AP, Silva PO, Marta CB et al. Cateter Central de Inserção Periférica: contribuições para a enfermagem Oncológica. Rev Enferm Ufpe On Line. 2019;13(3):715-31. doi: 10.5205/1981-8963-v13i03a23605 8p715-731-2019

10. Souza RAP, Carmo TG. Risco de infecção para o cliente oncológico em uso de cateter venoso central totalmente implantado. R. Epidemiol e Control Infecção. 2017;7(4):273-83. doi: $10.17058 /$ reci.v7i4.9885

11. Zerati AE, Wolosker N, Luccia N, Puech-Leão P. Cateteres venosos totalmente implantáveis: histórico, técnica de implante e complicações. J Vasc Bras. 2017;16(2):128-39. doi: 10.1590/1677$\underline{5449.008216}$

12. Oliveira EB, Reis MA, Avelar TM, Vieira SC. Cateteres venosos centrais totalmente implantáveis para quimioterapia: experiência com 793 pacientes. Rev Col Bras Cir 2012;40(3):186-190. doi: 10.1590/S0100-69912013000300004

13. Boller CEP, Senna KMS, Teixeira RS, Goulart MC, Tura BR, Santos MS. Avaliação de custo-efetividade do cateterismo em veia jugular interna guiado por ultrassom no Sistema Único de Saúde brasileiro. Jornal Brasileiro de Economia da Saúde. 2019;11(2):1128. doi: 10.21115/jbes.v11.n2.p112-8 
14. Mohammed S, Chhabra S, Bhatia PK, Kamal M, Bihani P. Quebra acidental da agulha durante cateterização da veia subclávia: uma adversidade indesejada!. Rev Bras Ter Int. 2017;29(3):397-398. doi: 10.5935/0103-507x.20170056

15. Kornbau C, Lee KC, Hughes GD, Firstenberg MS. Central line complications. Int J Crit IIIn Inj Sci. 2015;5(3):170-8. doi: 10.4103/2229-5151.164940

16. Neves Junior MA, Melo RC, Goes Junior AMO, Protta TR, Almeida CC, Fernandes AR et al. Infecções em cateteres venosos centrais de longa permanência: revisão da literatura. J. Vasc Bras. 2010;9(1):46-50. doi: 10.1590/S1677-54492010000100008

17. Cardoso DH, Mortola LA, Arrieira ICO. Terapia subcutânea para pacientes em cuidados paliativos: a experiência de enfermeiras na atenção domiciliar. J Nurs Health. 2016;6(2):34654. doi: $10.15210 /$ jonah.v6i2.6478

18. Secretaria Municipal de Gestão e Controle. Departamento de Controle Preventivo. Prefeitura Municipal de Campinas. Protocolo de Assistência de Enfermagem. Serviço de Atendimento Domiciliar em Campinas. [Internet]. 2015. Disponível em: http:// www.campinas.sp.gov.br/sa/impressos/adm/FO1236.pdf

19. Ercole FF, Macieira TGR, Wenceslau LCC, Martins AR, Campos CC, Chianca TCM. Revisão integrativa: evidências na prática do cateterismo urinário intermitente/demora. Rev Latino-Am Enfermagem. 2013;21(1):459-68. doi: 10.1590/S010411692013000100023

20. Almeida MM, Silva FWT, Lindoso AM, Pessoa RMC. Evidências na prática do cateterismo urinário. Rev Pre Infec e Saúde. 2015;1(4):52-62.

21. Brondani CM, Ramos LH, Beuter M, Lampert MA, Seiffert MA, Bruinsma JL. Caracterização de pacientes dependentes de tecnologias de um serviço de internação domiciliar. Rev Enferm Ufsm. 2013;3(esp.):689-9. doi: 10.5902/2179769211063

22. Carnaúba CMD, Silva TDA, Viana JF, Alves JBN, Andrade NL, Trindade Filho EM. Caracterização clínica e epidemiológica dos pacientes em atendimento domiciliar na cidade de Maceió, AL, Brasil. Rev Bras Geriatr Gerontol. 2017;20(3):352-62. doi: 10.1590/1981-22562017020.160163

23. Thompson A. Ventilação Mecânica domiciliar - Uma realidade cada vez mais frequente. Pulmão RJ. 2015;24(3):49-53.

24. Sociedade Brasileira de Nutrição Parenteral e Enteral. Terapia nutricional domiciliar. Rev Assoc Med Bras. 2012;58(4):408-11. doi: 10.1590/S0104-42302012000400008

25. Matos DFB. Análise do uso de antibióticos em pacientes internados em "home care" em Salvador (Bahia, Brasil).

[monografia]. Salvador: Universidade Federal da Bahia; 2014. 